Louisiana State University

LSU Digital Commons

Faculty Publications

Department of Biological Sciences

$1-1-2014$

Sexual differences of imprinted genes' expression levels

Mohammad Faisal

Louisiana State University

Hana Kim

Louisiana State University

Joomyeong Kim

Louisiana State University

Follow this and additional works at: https://digitalcommons.Isu.edu/biosci_pubs

Recommended Citation

Faisal, M., Kim, H., \& Kim, J. (2014). Sexual differences of imprinted genes' expression levels. Gene, 533 (1), 434-438. https://doi.org/10.1016/j.gene.2013.10.006

This Article is brought to you for free and open access by the Department of Biological Sciences at LSU Digital Commons. It has been accepted for inclusion in Faculty Publications by an authorized administrator of LSU Digital Commons. For more information, please contact ir@lsu.edu. 
Published in final edited form as:

Gene. 2014 January 1; 533(1): . doi:10.1016/j.gene.2013.10.006.

\title{
Sexual differences of imprinted genes' expression levels
}

\author{
Mohammad Faisal, Hana Kim, and Joomyeong Kim \\ Department of Biological Sciences, Louisiana State University, Baton Rouge, LA 70803, USA
}

\begin{abstract}
In mammals, genomic imprinting has evolved as a dosage-controlling mechanism for a subset of genes that play critical roles in their unusual reproduction scheme involving viviparity and placentation. As such, many imprinted genes are highly expressed in sex-specific reproductive organs. In the current study, we sought to test whether imprinted genes are differentially expressed between the two sexes. According to the results, the expression levels of the following genes differ between the two sexes of mice: Peg3, Ziml, Igf2, H19 and Zacl. The expression levels of these imprinted genes are usually greater in males than in females. This bias is most obvious in the developing brains of 14.5-dpc embryos, but also detected in the brains of postnatal-stage mice. However, this sexual bias is not obvious in 10.5-dpc embryos, a developmental stage before the sexual differentiation. Thus, the sexual bias observed in the imprinted genes is most likely attributable by gonadal hormones rather than by sex chromosome complement. Overall, the results indicate that several imprinted genes are sexually different in terms of their expression levels, and further suggest that the transcriptional regulation of these imprinted genes may be influenced by unknown mechanisms associated with sexual differentiation.
\end{abstract}

\section{Keywords}

Genomic imprinting; Sexual dimorphism; Peg3; Igf2; Zacl

\section{Introduction}

In mammals, a subset of genes are functionally different between two alleles due to an epigenetic mechanism called genomic imprinting, by which an allele of a given gene is repressed (imprinted) depending upon its parental origin (Bartolomei and Ferguson-Smith, 2011). About 100 autosomal genes are known to be imprinted, and these imprinted genes are usually expressed in early-stage embryos, placenta and brains (Bartolomei and FergusonSmith, 2011). According to the results from human and mouse genetic studies, mutations on these imprinted genes have very similar functional outcomes, such as changes in fetal growth rates and perturbations in animal behaviors that are associated with reproduction and social interaction (Bartolomei and Ferguson-Smith, 2011). Genomic imprinting is also found only in eutherian mammals, which have a very unusual reproduction scheme that involves viviparity and placentation (Renfree et al., 2013). Thus, genomic imprinting is believed to have co-evolved with this unusual reproduction scheme of placental mammals to control the dosage of a subset of genes that play critical roles in the reproduction-related physiology and

(c) 2013 Elsevier B.V. All rights reserved.

*Corresponding author. Tel.:+1 225578 7692; fax: +1 225578 2597. jkim@ @lsu.edu (J. Kim).

Conflict of interest statement

The authors declare no conflict of interest.

Appendix A. Supplementary data

Supplementary data to this article can be found online at http://dx.doi.org/10.1016/j.gene.2013.10.006. 
behaviors (Feil and Berger, 2007; Ivanova and Kelsey, 2011; Keverne, 2013; Renfree et al., 2013).

In mammals, sex is determined first by chromosome, more specifically by the testisdetermining factor on Y chromosome (Sry), which is responsible for the formation of two different reproductive organs, testis and ovary. The subsequent sexual differentiation of developing brains is further triggered by the high levels of testosterone released from neonate's testis, which eventually determines animal sexual behaviors for reproduction (McCarthy and Arnold, 2011). As an outcome of a series of sexual determination and differentiation, males and females display quite different morphological and behavioral traits although both have almost identical genome sequences. Recent surveys further demonstrate that a large number of autosomal genes are sexually biased or dimorphic in terms of their expression levels in various tissues (Mozhui et al., 2012; van Nas et al., 2009; Yang et al., 2006). As described earlier, the overall biological impetuses of genomic imprinting are closely associated with the reproduction strategy of eutherian mammals, and thus it is likely that some of imprinted genes might also be sexually biased or dimorphic in terms of their function. In that regard, it is interesting to note that many imprinted genes are known to control fetal growth rates, variations in which could easily lead to different body sizes, a well-known sexual dimorphism in mammals. Also, many imprinted genes are highly expressed in the hypothalamus, which is one of the main targets within brains that are differentially organized by gonadal hormones between the two sexes (McCarthy, 2009; McCarthy and Arnold, 2011). Thus, it is feasible to predict that genomic imprinting may interact functionally with sexual differentiation for the successful reproduction of eutherian mammals.

According to recent studies, some of imprinted genes are indeed either controlled by or at least affected by sexual differentiation although it is currently unknown at what level and to what extent sexual differentiation influences the function of these genes. During the gestation period of the mouse, the expression levels of several imprinted genes were shown to respond dramatically to a temporary mal-nutrition condition (starvation), and yet these responses were sex-specific. In particular, the expression level changes of Peg3 were detected only in males, but not in females, whereas those of Zacl and Igf2 were more pronounced in females (Radford et al., 2012). Similarly, a mutagenesis experiment deleting the imprinting control region of the Peg3 domain, Peg3-DMR (Differentially Methylated Region), also derived unusual sex-specific effects: male pups tend to be more severely affected by the deletion than female pups in terms of body weight reduction and embryonic lethality (Kim et al., 2012). In the current study, we performed a series of expression analyses using various-stage mouse tissues to measure to what extent genomic imprinting is influenced functionally by sexual determination and differentiation. According to the results, several imprinted genes are indeed variable between two sexes in terms of their expression levels in developing brains.

\section{Materials and methods}

\subsection{Mouse strain and breeding}

The mouse strain with B6/129-mixed background mice was obtained from the Jackson lab and has been maintained at the animal care facility of Louisiana State University according to the animal care protocol. All the experiments related to mice were performed in accordance with the National Institutes of Health guidelines for care and use of animals, and also approved by the Louisiana State University Institutional Animal Care and Use Committee (IACUC), protocol \#10-071. All animals were kept in a temperature-controlled room $\left(22^{\circ} \mathrm{C}\right)$ with $3-4$ mice per cage at a 12-hour light/dark cycle and they were given $\mathrm{ad}$ 
libitum access to food and water. Embryos with different developmental stages were harvested through time-mated breeding schemes.

\subsection{Sex determination of embryos}

Genders of embryos were determined by PCR using the Maxime PCR premix kit (Intron Biotech). The Primer set was mouse Sry gene; mSry-F (5GTCCCGTGGTGAGAGGCACAAG-3) and mSry-R (5-GCAGCTCTACTC CAGTCTTGCC-3). The PCR conditions were 35 cycles at $95^{\circ} \mathrm{C}$ for $30 \mathrm{~s}, 60^{\circ} \mathrm{C}$ for $30 \mathrm{~s}$ and $72{ }^{\circ} \mathrm{C}$ for $30 \mathrm{~s}$. Genomic DNA was prepared from the amnion sac of harvested embryos. Each tissue was incubated overnight at $65^{\circ} \mathrm{C}$ in the lysis buffer $(0.1 \mathrm{M}$ Tris-Cl, $5 \mathrm{mM}$ EDTA, $0.2 \%$ SDS, $0.2 \mathrm{M} \mathrm{NaCl}, \mathrm{pH} 8.0,20 \mu \mathrm{g} / \mathrm{ml}$ Proteinase K). This lysed extract was diluted 30 times with distilled water and $1 \mu \mathrm{l}$ of the diluted extract was used in each PCR reaction.

\subsection{RNA isolation and quantitative RT-PCR analyses}

Total RNA was isolated using a commercial kit Trizol (Invitrogen) as per the manufacturer's protocol. The isolated total RNA $(5 \mu \mathrm{g})$ from each of the samples was reverse-transcribed using M-MLV kit (Invitrogen). The subsequent cDNA was used as a template for quantitative real time PCR. This analysis was performed using the iQ SYBR Green Supermix (Bio-Rad) using the iCycler iQTM multicolor real-time detection system (BioRad). All qRT-PCR reactions were carried out for 40 cycles under the standard PCR conditions and two genes ( $\beta$-actin and 28S) were used as the internal control. Results of the qRT-PCR were analyzed based on the threshold $(\mathrm{Ct})$ value. The experiments were performed in triplicate for each imprinted gene. At first a $\Delta \mathrm{Ct}$ value was calculated through subtracting the $\mathrm{Ct}$ value of that given replicate of a particular gene from the average $\mathrm{Ct}$ value of the internal control ( $\beta$-actin). After that, the fold difference was determined for each replicate by raising 2 to the $\Delta \Delta \mathrm{Ct}$ powers (Winer et al., 1999). The relative expression level of an individual sample was further calculated through dividing its raw value with the average raw value of a given litter. One-tailed Student's $t$-test was used for statistical comparison of four male and four female samples $(\mathrm{p}<0.05)$. Primer sequences and PCR conditions are available as Supplemental Table 3.

\subsection{Box plot and data analysis}

Box plot (also known as box and whisker diagram) was used to present the groups of numerical values in the current study. The data obtained from qRT-PCR was shown in their five number summaries, such as lower quartile (Q1), median $(\mathrm{Q} 2)$, upper quartile $(\mathrm{Q} 3)$, smallest observation (sample minimum), and largest observation (sample maximum). In this graphical representation, the first segment of the stacked column is invisible and ends where the lower boundary of the second quartile begins. The next segment consists of second quartile, which is calculated as: median - first quartile. The third segment is the third quartile and calculated as: third quartile - median. The maximum and minimum values are represented by the length of the whiskers and calculated as: first quartile - minimum and maximum - third quartile, respectively.

\section{Results}

\subsection{Expression level differences of imprinted genes in $14.5-\mathrm{dpc}$ fetuses}

To detect potential sexual differences associated with genomic imprinting, we decided to measure expression level differences of imprinted genes between the two sexes of mice. Imprinted genes are usually expressed in brains and placenta during embryonic stages (Bartolomei and Ferguson-Smith, 2011), and thus we harvested two litters of 14.5-dpc (days post coitum) fetuses that had been derived from the mouse strain with 129/B6-mixed 
background. The gender of each fetus was first determined with PCR using DNA isolated from the amnion sac. From each litter, two males and females were selected for the isolation of total RNA from embryo head and placenta. The isolated RNA was individually reversetranscribed and used for quantitative PCR analyses. Two internal controls ( $\beta$-actin and 28S) were used for normalization of the expression level of each imprinted gene. The relative expression levels of a given gene between individual embryos were represented as percentile scores by dividing each embryo's expression value with the average value of a given litter (Table 1). Two values were further calculated from the initial set of percentile scores: F/M ratio (the ratio of female to male values) and STD ratio (the ratio of standard deviation values of the entire set to each gender group). The actual formula for the STD ratio is as follows: the standard deviation value of the entire set divided by the average value of two standard deviation values from female and male groups. The STD ratio was used to evaluate the degree to which gender difference is accountable for the overall variation in a given gene: the greater values indicate the variations driven more by gender difference rather than stochastic individual differences.

The expression levels of 15 imprinted genes and one non-imprinted gene (Esrl, estrogen receptor 1) were initially analyzed using the total RNA isolated from the embryos and placentas of two harvested litters (Supplemental material 1). As expected, the majority of imprinted genes displayed high levels of expression in both embryo head and placenta based on the $\mathrm{Ct}$ (threshold cycle) values: the $\mathrm{Ct}$ values of imprinted genes ranged from 15 to 25 while the $\mathrm{Ct}$ values of the two internal controls were 14 through 15 . Out of the initial set of imprinted genes tested, the following 5 genes displayed consistently different levels of expression between two sexes in the head of 14.5-dpc embryos: Peg3, Zim1, Igf2, H19 and Zacl (Table $1 \&$ Fig. 1A). The expression levels of these genes were greater in males than in females with the F/M ratio ranging from 0.242 to 0.552 . The observed differences were statistically significant based on the results from Student's $t$-test with the p values ranging from 0.0004 to 0.008 . The STD ratios of these 5 genes were around 2 while the values of the remaining imprinted genes without any gender bias were around 1 . This further supports this initial conclusion that gender difference is the main factor contributing to the overall variations observed in each of these genes. Two additional genes, Usp29 and Gtl2, also showed somewhat biased expression levels (Supplemental material 2), but require further investigation in the near future. The expression levels of these 5 genes were also analyzed in placenta (Table $1 \&$ Fig. 1B). Among the 5 genes, two genes (Peg3 and H19) displayed different expression levels between two sexes with the F/M ratios being 0.617 for Peg3 and 0.539 for $\mathrm{H1}$. However, the difference observed only from $\mathrm{Peg} 3$ was statistically significant $(\mathrm{p}=0.0054)$, which was also supported by the STD value of Peg3 (2.135). Overall, this series of analyses concluded that the expression levels of the 5 genes (Peg3, Zim 1, Igf2, H19, Zacl) differ between two sexes. In placenta, the expression levels of Peg3 also showed a difference between two sexes.

\subsection{Expression level differences of imprinted genes in 10.5-dpc embryos}

The sexual differences of expression level of the 5 imprinted genes were further analyzed using different developmental-stage embryos. During mouse development, sexual differentiation commences around embryonic day 12.5, and thus any sexual differences prior to the 12.5-dpc stage is regarded as an outcome of biological events stemming from different sex chromosomes (McCarthy and Arnold, 2011). Given this reasoning, we harvested two litters of 10.5-dpc embryos derived from the breeding of the 129/B6-mixed strain: the first set with one male and two females and the second set with two males and one female. Another series of expression analyses were performed using these six embryos in a similar manner as the 14.5-dpc embryo set (Fig. 2). According to the results, three genes (Peg3, $I g f 2, H 19)$ displayed greater expression levels in males than in females, but the observed 
differences in these genes were not statistically significant $(p=0.2,0.23,0.074$, respectively). The STD ratios of these three genes were also close to 1, suggesting that gender difference was not a main factor contributing to the overall variations for these genes (Supplemental material 2). Thus, this series of expression analyses concluded that the expression levels of these 5 genes are likely similar between the two sexes of 10.5-dpc embryos. This further suggests that the sexual differences of the 5 genes observed in the 14.5-dpc embryos may not be originated from any difference associated with sex chromosomes.

\subsection{Expression level differences of imprinted genes in neonates' brain and liver}

The sexual differences of expression level of the 5 genes were also analyzed using the tissues derived from neonates. Mouse brains become sexually organized during late embryonic through postnatal stages (E18-P10) due to high levels of testosterone released from neonate testes (McCarthy, 2009; McCarthy and Arnold, 2011). Liver is also known to be sexually dimorphic, yet several imprinted genes, such as Igf2 and H19, are highly expressed in neonatal-stage livers (Bartolomei and Ferguson-Smith, 2011). Thus, we harvested two separate litters of neonates at postnatal day 5 (P5). The total RNA isolated from the brain and liver of these neonates was analyzed in a similar scheme as the above (Fig. 3). For this series of analyses, two additional genes, Adamts2 (ADAM metallopeptidase with thrombospondin type 1 motif, 2) and Prlr (Prolactin receptor), were also included as controls, where Adamts 2 is male specific and Prlr is female specific. The expression levels of Adamts 2 and Prlr are known to be sexually biased: greater levels in males for Adamts 2 versus greater levels in females for Prlr (Mozhui et al., 2012). In brain, according to the results, the expression levels of all 5 genes are greater in males than in females. However, the observed differences were statistically significant only in the case of two genes (Igf2 and H19). This was also supported by the STD ratios (2.135 for Igf 2 and 1.707 for 119 ), indicating that the variations observed from these two genes were driven by gender difference. As expected, the two control genes (Adamts 2 and Prlr) showed greater levels in males and in females, respectively. In the liver, the expression levels of the 5 genes were also somewhat greater in males than in females, yet the observed variations appeared to be driven more by individual differences rather than gender differences based on the STD ratios (around 1). Consistent with this, this difference was not also statistically significant. Overall, this series of analyses concluded that the expression levels of two genes (Igf2 and H19) are sexually different in the brains of neonates. The observed differences of these two genes are also similar to those observed in the heads of the 14.5-dpc embryos: greater expression levels in males than in females.

\section{Discussion}

In the current study, we performed a series of expression analyses to test whether the expression levels of imprinted genes differ between the two sexes of mice. According to the results, the expression levels of five genes (Peg3, Ziml, Igf2, H19, Zacl) in the head of 14.5 -dpc embryos differ between the two sexes. This difference is not obvious at the 10.5dpc stage of mouse development. In contrast, the expression levels of two genes (Igf2 and H19) are still sexually different in the brain of neonates. Overall, these results suggest that the transcription of a subset of imprinted genes may be influenced by sexual differentiation in the brain of developing embryos.

Out of the 15 imprinted genes tested, five imprinted genes are sexually different in terms of their expression levels (Table $1 \&$ Fig. 1). This is somewhat consistent with recent findings that approximately $10 \%$ of mammalian genes are sexually biased in terms of their expression levels (Mozhui et al., 2012; van Nas et al., 2009; Yang et al., 2006). This 
suggests that genomic imprinting may also be influenced by some unknown mechanisms associated with sexual differentiation. It is, however, important to note that the sexual difference observed in the imprinted genes is somewhat unique: the expression levels of these imprinted genes are all greater in males than in females. This may be related to one of well-known sexual dimorphisms in mammals, a bigger body size in males than in females. On the other hand, one of the main biological impetuses of genomic imprinting is controlling fetal growth rates to cope with an unusual reproduction scheme of eutherian animals (Renfree et al., 2013). Consistent with this, the majority of imprinted genes are indeed involved in controlling fetal growth rates although the protein functions of imprinted genes are very diverse, including growth factors, growth factor receptors, enzymes and DNA-binding proteins. Thus, the sexual difference associated with a subset of imprinted genes displaying greater levels of expression in males may be an outcome of unknown transcriptional programs that are designed to derive different body sizes between the males and females of mammals.

Developmental profiling of the sexual difference associated with imprinted genes provides unique insights regarding when and how this sexual bias is established (Figs. 1-3). The sexual difference is not obvious at the 10.5-dpc stage, suggesting that the observed sexual difference may not be originated from any genetic difference associated with sex chromosomes. On the other hand, the sexual difference is most prominent in the brains of 14.5-dpc embryos. The expression levels of two genes (Igf2 and H19) are also sexually different at early postnatal stages (P5), a time point when developing brains are sexually organized by the high levels of testosterone released from the neonate's testes. However, since the two imprinted genes are already sexually different at the 14.5 -dpc stage, the sexual difference observed at the P5 stage may not be an outcome of this sexual organization of developing brains. Instead, the sexual difference observed from the imprinted genes is likely established at earlier stages but later than the 10-dpc stage of development, and this established difference may be maintained in the two genes (Igf2 and H19), thus visible at the P5 stage. This possibility is further supported by the fact that many imprinted genes are highly expressed in the areas of brain other than the hypothalamus, a main target that is known to be sexually organized by a sudden surge of testosterone at postnatal stages (McCarthy, 2009). In conclusion, the sexual difference observed at the neonatal stage may not be an outcome of the testosterone-driven sexual organization of developing brain. Instead, the sexual difference associated with the imprinted genes is most likely established at much earlier stages during brain development.

\section{Supplementary Material}

Refer to Web version on PubMed Central for supplementary material.

\section{Acknowledgments}

We would like to thank Muhammad Ekram, Wesley Frey, An Ye, Pinithi Perera, Arundhati Bakshi, Corey Bretz and Hongzhi He for their careful reading and discussion of the manuscript. This research was supported by the National Institutes of Health (J.K. R01-GM066225 and R15-ES019118).

\section{Abbreviations}

$\begin{array}{ll}\text { Peg3 } & \text { paternally expressed gene } 3 \\ \text { Zim1 } & \text { zinc finger, imprinted 1 } \\ \text { Usp29 } & \text { ubiquitin carboxyl-terminal hydrolase } 29\end{array}$


Zac1 zinc finger protein 1 regulating apoptosis and cell cycle arrest

Igf2 insulin-like growth factor 2

Grb10 growth factor receptor-bound protein 10

Dlk1

Snrpn

protein delta homolog 1 isoform 1 precursor

Ube3a

small nuclear ribonucleoprotein-associated

Ndn

ubiquitin-protein ligase E3A isoform 2

Nnat

necdin

Rasgrf1

neuronatin isoform alpha

Esr1

ras-specific guanine nucleotide-releasing factor

Adamts2 estrogen receptor 1

preproprotein

Prlr prolactin receptor precursor

Mest mesoderm-specific transcript protein

H19 H19 mRNA

Gt12 gene target locus 2

\section{References}

Bartolomei MS, Ferguson-Smith AC. Mammalian genomic imprinting. Cold Spring Harb. Perspect. Biol. 2011; 3:a002592. [PubMed: 21576252]

Feil R, Berger F. Convergent evolution of genomic imprinting in plants and mammals. Trends Genet. 2007; 23:192-199. [PubMed: 17316885]

Ivanova E, Kelsey G. Imprinted genes and hypothalamic function. J. Mol. Endocrinol. 2011; 47:R67R74. [PubMed: 21798993]

Keverne EB. Importance of the matriline for genomic imprinting, brain development and behaviour. Philos. Trans. R. Soc. Lond. B Biol. Sci. 2013; 368:20110327. [PubMed: 23166391]

Kim J, et al. Imprinting control region (ICR) of the Peg3 domain. Hum. Mol. Genet. 2012; 21:26772687. [PubMed: 22394678]

McCarthy MM. The two faces of estradiol: effects on the developing brain. Neuroscientist. 2009; 15:599-610. [PubMed: 19700741]

McCarthy MM, Arnold AP. Reframing sexual differentiation of the brain. Nat. Neurosci. 2011; 14:677-683. [PubMed: 21613996]

Mozhui K, Lu L, Armstrong WE, Williams RW. Sex-specific modulation of gene expression networks in murine hypothalamus. Front. Neurosci. 2012; 6:63. [PubMed: 22593731]

Radford EJ, et al. An unbiased assessment of the role of imprinted genes in an intergenerational model of developmental programming. PLoS Genet. 2012; 8:e1002605. [PubMed: 22511876]

Renfree MB, Suzuki S, Kaneko-Ishino T. The origin and evolution of genomic imprinting and viviparity in mammals. Philos. Trans. R. Soc. Lond. B Biol. Sci. 2013; 368:20120151. [PubMed: 23166401]

van Nas A, et al. Elucidating the role of gonadal hormones in sexually dimorphic gene coexpression networks. Endocrinology. 2009; 150:1235-1249. [PubMed: 18974276]

Winer J, Jung CK, Shackel I, Williams PM. Development and validation of real-time quantitative reverse transcriptase-polymerase chain reaction for monitoring gene expression in cardiac myocytes in vitro. Anal. Biochem. 1999; 270:41-49. [PubMed: 10328763] 
Yang X, et al. Tissue-specific expression and regulation of sexually dimorphic genes in mice. Genome Res. 2006; 16:995-1004. [PubMed: 16825664] 

A) $14.5-\mathrm{dpc} \mathrm{Head}$

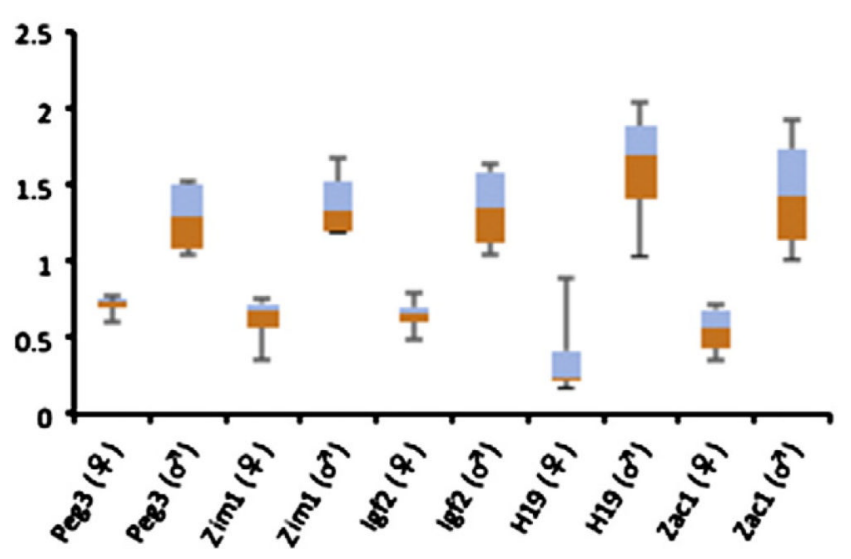

B) 14.5-dpc Placenta

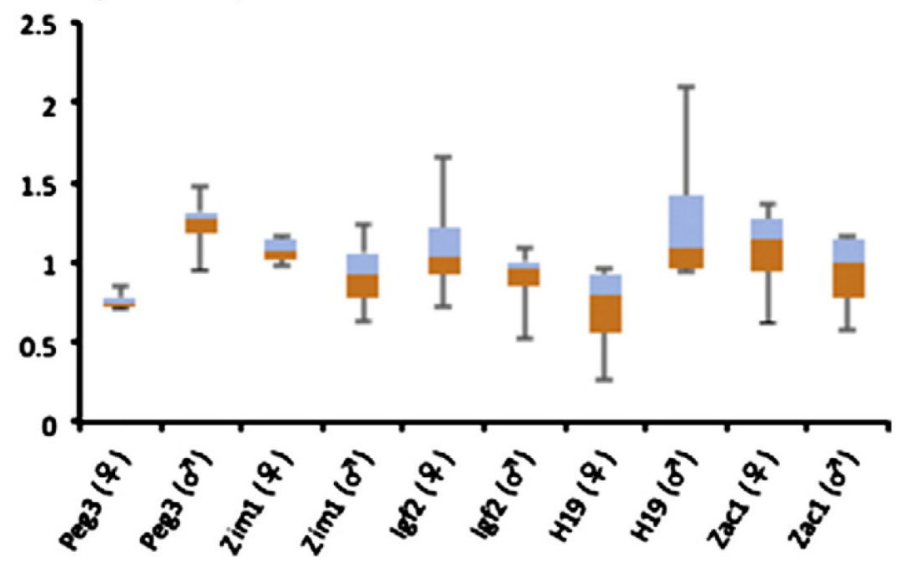

Fig. 1.

Sexual difference of expression level of imprinted genes in the 14.5-dpc fetuses. Expression levels of imprinted genes are compared between the two sexes of mice using the total RNA isolated from the embryo head (A) and placenta (B) of 14.5-dpc fetuses. Relative expression level is shown as box plot in their five number summaries such as lower quartile (Q1), median (Q2), upper quartile (Q3), smallest observation (sample minimum), and largest observation (sample maximum). Total of 8 mice for both male and female were used for this analysis. The individual values used for these graphs are presented in Table 1. 


\section{5-dpc Embryo}

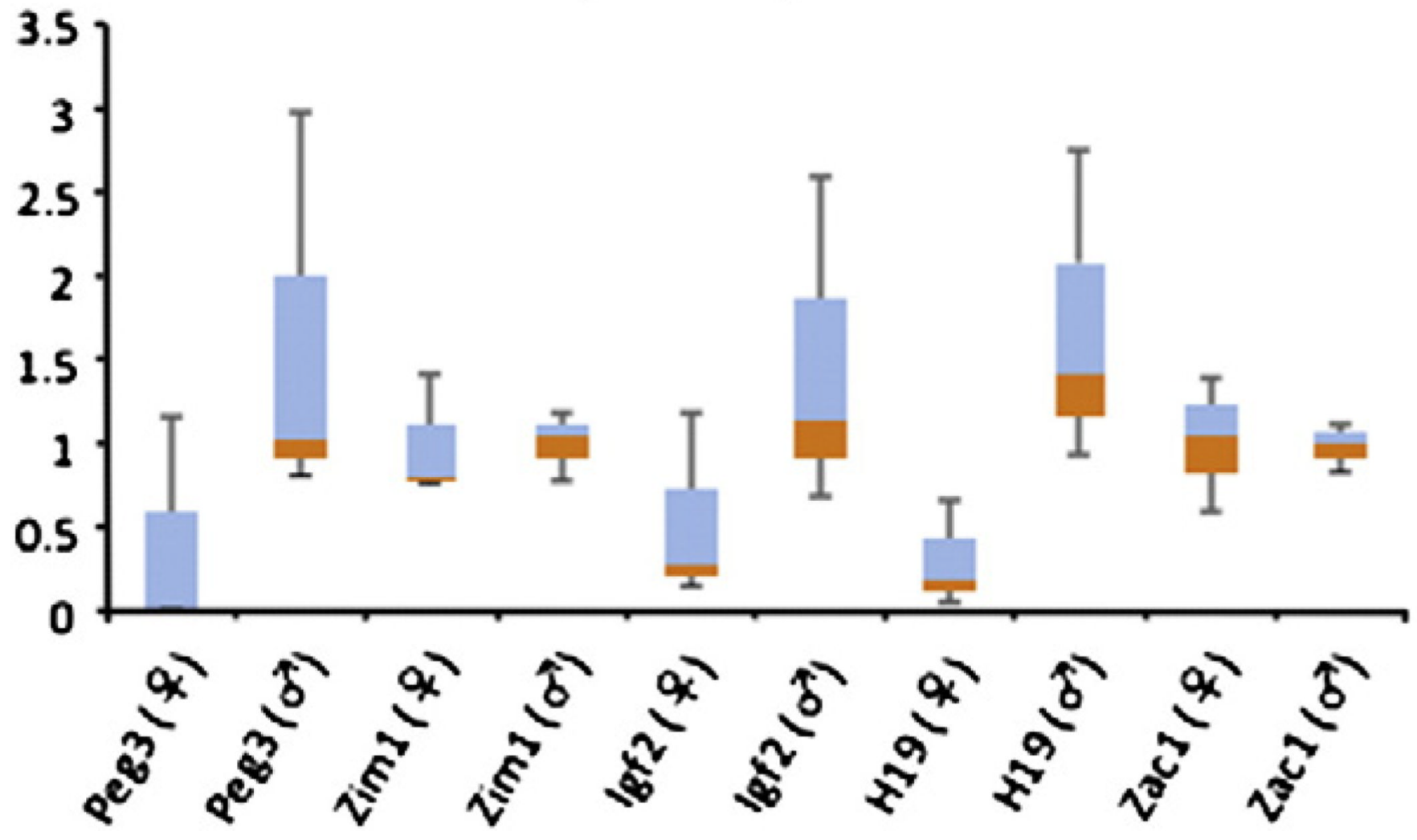

Fig. 2.

Sexual difference of expression level of imprinted genes in the 10.5-dpc embryos.

Expression levels of imprinted genes are compared between the two sexes of 10.5-dpc embryos. This series of analyses used the total RNA isolated from the entire body of total 6 embryos. Box plot and statistical analysis do not show any significant differences in any of these five imprinted genes' expression level between males and females although the relative expression level of H19 is somewhat different and seem to be higher in male, still not statistically significant enough. The individual values used for these graphs are presented in Supplemental Table 2. 


\section{A) P5 Brain}

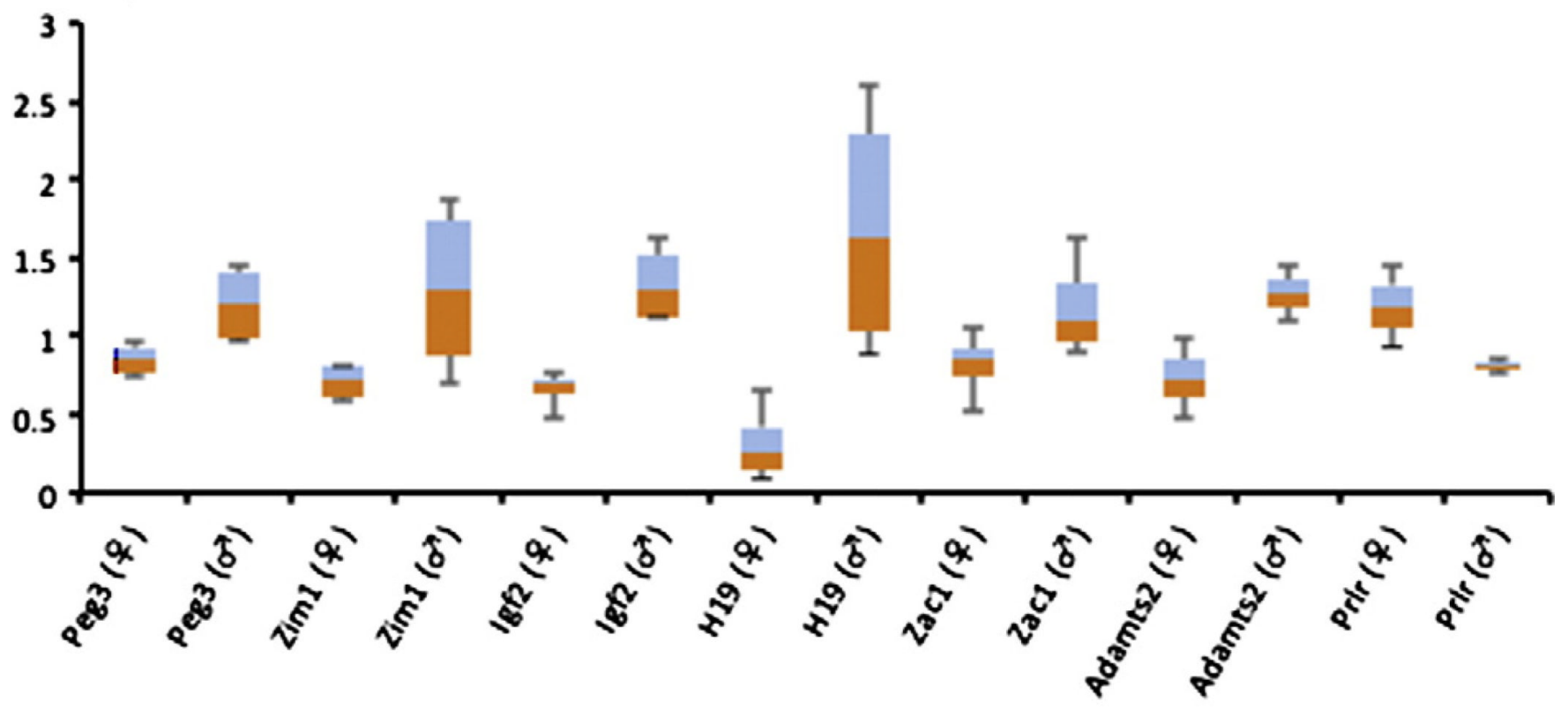

B) P5 Liver

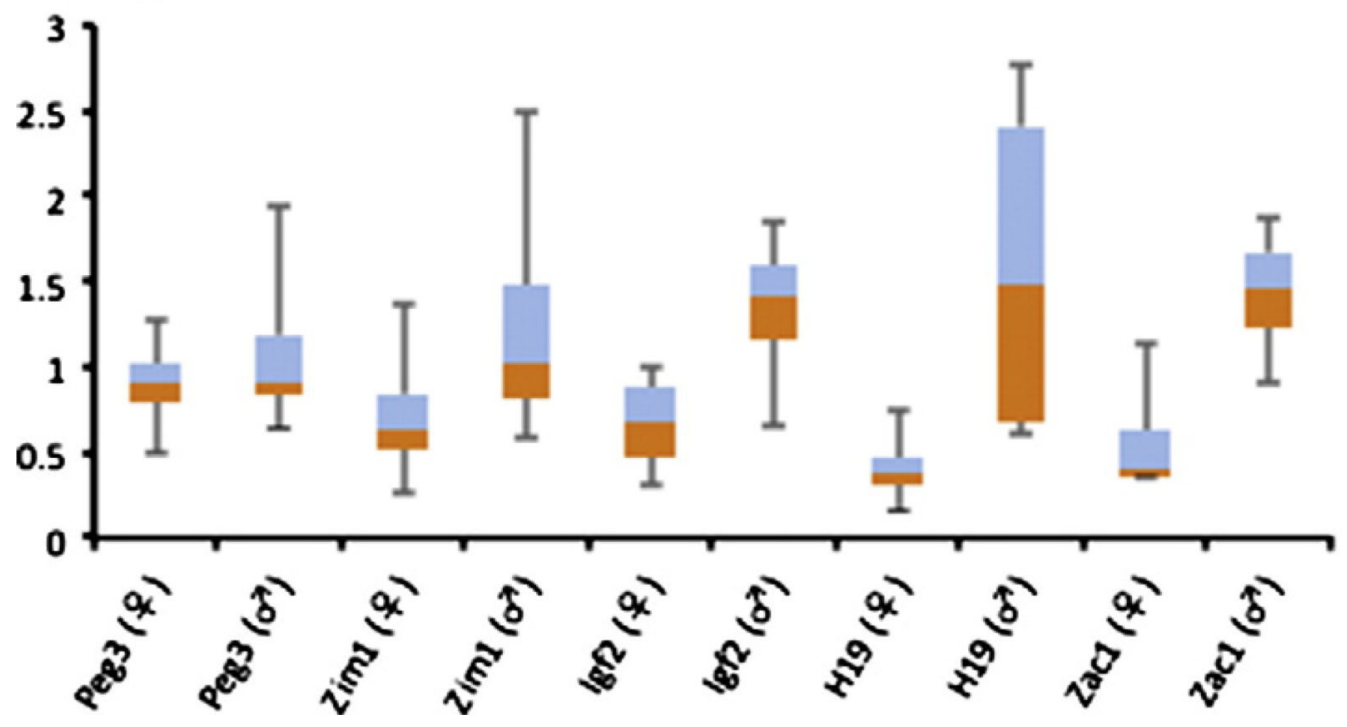

Fig. 3.

Sexual difference of expression level of imprinted genes in the neonates of postnatal day 5 (P5). Expression levels of imprinted genes are compared between the two sexes of neonates using the total RNA isolated from brains (A) and livers (B). This analysis used a total of 8 neonates for both male and female. Two genes (Adamps2 and Prlr) with male- or femalebiased expression levels, respectively, were also included in the analyses of brain samples. The individual values used for these graphs are presented in Supplemental Table 2. 
